\title{
Tailoring interventional arrhythmia therapy to individual patients: seeking the Art and the Science of Cardiac Electrophysiology in 2016
}

\author{
Sanjeev Saksena ${ }^{1,2}$
}

Received: 3 January 2016 / Accepted: 3 January 2016 /Published online: 30 January 2016

(C) Springer Science+Business Media New York 2016

2015 was a tumultuous year throughout the globe and a time of continuing and rapid change in the world of medicine as well. The Journal weathered this period with stability and showed steady growth as we continue to seek new horizons of scientific endeavor and critically examine existing ones.

Nevertheless, the start of a new year promotes reflection and reassessment. The direction of evolution of our field remains an ongoing subject for critical examination in this commentator's view. In prior columns, I have focused on these changes, including the paradigm shifts occurring in medical care and their myriad Achilles heels [1,2]. As we have pursued better care for our patients with evidence based guidelines, the overall shift to standardized care has promoted consideration of and access to superior care. These guidelines have become the key underpinning of an enormous health care delivery system and industry, used to drive quality, cost and eventually access to care.

However, the limitations of a broad strokes universal approach to therapy selection have also become painfully apparent. For example, there is increasing recognition of the impact of co-morbidities on arrhythmia outcomes and the inability of many patients to benefit from the prescribed treatment or respond in the expected manner. At the other extreme, the value of early antiarrhythmic interventions in disease states to prevent arrhythmia progression is being appreciated, even as too many patients fail to obtain access to modern antiarrhythmic

Sanjeev Saksena

cmenj@aol.com

1 Rutgers' - The Robert Wood Johnson Medical School, Piscataway, NJ, USA

2 The Electrophysiology Research Foundation, Warren, NJ, USA therapy. The guidelines have helped with the latter in substantial measure but the former remains a challenge.

In 2014, I focused on the arrhythmia substrate and intervention interactions as one such element in individual patient outcomes [3]. New advances in imaging technology now allow us to visualize the former and to better target the latter. However the individual response of a patient to therapy remains often an unpredictable variable. Nowhere is this more apparent than in the experience with catheter ablation of atrial fibrillation. The last decade has been occupied with a vigorous debate on this therapy, focusing on its proper application and thoughtful withholding. In 2010, this Journal column spoke of a "new view from the summit," wherein translational science and genomics would become applied clinical therapeutics in interventional electrophysiology [4]. This is now a reality.

In this issue of the Journal, Mohanty and coworkers in a breakthrough report analyzed novel single nucleotide polymorphisms (SNPs) in patients with atrial fibrillation undergoing catheter ablation. They studied their association with mapped non-pulmonary vein triggers and atrial scar and their relationship to the observed outcomes of this treatment [5]. In suggesting that these SNPs may relate to the observations in the clinical laboratory, their insights may help reconcile the many and varied electrophysiologic and disease state findings associated with this arrhythmia. In a prescient editorial, Huang and Darbar place these new observations in perspective, and how they can potentially fit into the embryology and development of the arrhythmia substrate as well as the electrophysiology of sinoatrial and atrial arrhythmias [6].

This returns us to our original theme, the individuality of a patient and that guidelines of care significantly lag expanding scientific knowledge. Perhaps, the art of medicine lies in recognizing the former and the limitations of the latter. For those involved in enforcing rigid paradigms of health care delivery today, this would be a lesson in scientific humility. The decade 
long strident debate on how to best treat atrial fibrillation with differing catheter ablation methods, or even other unconventional modalities such as dual site right atrial pacing, should take pause and assimilate new knowledge.

In another context, Albert Einstein critically wrote "It has become appallingly obvious that our technology has exceeded our humanity." Unfortunately, all too often patient satisfaction with health care delivery today is mirrored in the comment. In the future, technology could lead us to the very human profile of the individual patient, perhaps tailoring interventional therapy once more to the individual patient.

The Journal continued to thrive in 2015. Our manuscript submission rate continues to increase now being almost exclusively focused on original articles and reviews. In the coming year, we plan to bring two special issues led by our Associate Editors, focused on areas of enormous current interest. Drs. Leonardo Calo and Riccardo Cappato are coediting a special Journal issue on "Inappropriate Sinus Tachycardia" highlighting advances in pathophysiology and therapy this quarter. Dr. Fred Kusumoto will be presenting our second special issue on Health Policy in 2016 later this year. Our assistant editor team continues our online presence with Twitter and Facebook posts, reaching ever further across the globe. Our association with the European Cardiac Arrhythmia Society remains robust, with an increasingly visible annual scientific session and abstract presentations, with the annual Journal abstract issue being highly downloaded.

Once again, it is my pleasure to recognize those who make this Journal's success possible. The team is led by our editor Lisa Aquilino and her staff, and supported by the editorial office at Springer and the firmament of our reviewers that allow us to present high level science to our readers. We recognize our top reviewers annually and they receive Journal subscriptions as an expression of our gratitude. My co-editors have my profound thanks for continuing their unflagging support of this endeavor.

Respectfully submitted on behalf of the editors

Sanjeev Saksena

Editor in Chief

Rutgers' - The Robert Wood Johnson Medical School, Piscataway, New Jersey

The Electrophysiology Research Foundation, Warren, New Jersey

\section{References}

1. Saksena, S. (2012). Clinical trials of antiarrhythmic therapies and optimizing health care resource deployment: the need for a paradigm shift. Journal of Interventional Cardiac Electrophysiology, 1, 1-3.

2. Saksena, S. (2013). Antiarrhythmic therapy - the paradigm shifts in 2012: will it be enough to result in improved health care policies? Journal of Interventional Cardiac Electrophysiology, 1, 1-3.

3. Saksena, S. (2014). Interactions of interventional antiarrhythmic therapies with individual diseases and their substrate: the next challenge in improving patient outcomes. Journal of Interventional Cardiac Electrophysiology, 1, 1-2.

4. Saksena, S. (2010). A truly new view from the summit: from translational science to outcomes research in clinical arrhythmology. Journal of Interventional Cardiac Electrophysiology, 1, 1-2.

5. Mohanty S, Hall P, Mohanty P et al. (2016). Novel association of polymorphic genetic variants with predictors of outcome of catheter ablation in atrial fibrillation: new directions from a prospective study (DECAF). Journal of Interventional Cardiac Electrophysiology, 45(1). doi:10.1007/s10840-015-0069-2

6. Huang H, Darbar D. (2016). Gene-guided therapy for catheterablation of atrial fibrillation: are we there yet? Journal of Interventional Cardiac Electrophysiology, 45(1). doi:10.1007/ s10840-015-0086-1 